\title{
Estimaite of Magnetic Forces on Beam Sweeping Kickers
}

PBAR Note 632

Martin $\mathrm{Hu}$

Feb. 23, 2000

The bean sweeping magnet kickers are two pairs of conductors placed 90 degrees apart inside a circular magnetic yoke. Each pair has the same axcitation curreent in the opposite directions, and the two currents are a sine and a cosine in phase.

To estimate the magnetic forces on the kickers due to the excitation currents, we make the following simplifications:

1. The four conductors are all parallel to one another.

2. There are no relative motions within the system.

3. the magnetic yoke has $\mu=\infty$, and the fields are zero near the outside inner surface of the yoke.

Fig. 1 shows the cross section of the conductors. Conductors 1 and 3 have equal and opposite currents, conductors 2 and 4 have equal and opposite currents, and the current $I_{1}$ leads $I_{2}$ by 90 degrees. We can write $I_{1}$ as $I_{\text {peak }} \cdot \sin \theta, I_{2}$ as $I_{\text {peak }} \cdot \cos (\theta)$, $I_{3}$ as $-I_{\text {peqk }} \cdot \sin (\theta)$, and $I_{4}$ as $-I_{\text {peak }} \cdot \cos (\theta)$, where $I_{\text {peak }}$ is 10,000 amps. We now wish to calcul ite the net forces on a conductor due to the other three conductors and the magnetic yoke.

We makt: the simplifying assumption that the magnetic permittivity, $\mu$, is infinite; in reality $\mu$ is about 60 and very little B-field escapes to the outside. Assuming that the B-field is zero right outside the yoke, we can then replace the yoke with 4 image currents in the same directions at a distance $a$ so that the B-field does cancel at the outer cirisumference of the yoke (Fig. 2). The problem is now reduced to summing up all the fcrces on one conductor due to the other 7 currents. As shown in Fig. 1, for example for conductor 1 , we decompose the forces on it into $F_{x}$ (radially outward) and $F_{y}$ ( angential). Then,

$F_{1 x}=F_{2 l(x)}+F_{31(x)}+F_{41(x)}+F_{1 i 1(x)}+F_{2 i 1(x)}+F_{3 i 1(x)}+F_{4 i 1(x)}=$

$\frac{\mu_{0} l}{2 \pi r} \cdot I_{\left.p e_{i}\right)^{\prime}}^{2} \cdot\left(-\sin \theta \cos \theta \sin \beta+\frac{1}{\sqrt{2}} \sin ^{2} \theta+\sin \theta \cos \theta \sin \beta+\right.$

$\left.\frac{r}{a} \sin ^{2} \theta-\frac{r}{b} \sin \theta \cos \theta \sin \alpha+\frac{r}{a+\sqrt{2} r} \sin ^{2} \theta+\frac{r}{b} \sin \theta \cos \theta \sin \alpha\right)$,

and 
$F_{1 y}=F_{21(y)}+F_{31(y)}+F_{41(y)}+F_{1 i 1(y)}+F_{2 i 1(y)}+F_{3 i 1(y)}+F_{4 i 1(y)}=$

$\frac{\mu_{0} l}{2 \pi r} \cdot I_{\text {pea }}^{2}: \cdot(-\sin \theta \cos \theta \cos \beta+0-\sin \theta \cos \theta \cos \beta+0-$

$\frac{r}{b} \sin \theta \cos \theta \cos \alpha+0-\frac{r}{b} \sin \theta \cos \theta \cos \alpha$ ),

where $\theta=\omega t$ is the reference phase angle of all currents, $l$ is the length of the conducter, $l=27.9 \mathrm{~cm}, r=2.00 \mathrm{~cm}, a=4.98 \mathrm{~cm}, b=6.55 \mathrm{~cm}, \beta=45^{\circ}$ and $\alpha=12.5^{\circ}$.

Then,

$F_{1 x}=1.37 \sin ^{2} \theta$

and

$F_{1 y}=-2.00 \sin \theta \cos \theta$

where a positive $F_{x}$ indicates an radially outward force, and a positive $F_{y}$ indicates a force causing a counter-clockwise rotation. Similar analyses for the other three conductérs yield

$F_{1 x}=F_{3 x}=1.37 \sin ^{2} \theta$,

$F_{2 x}=F_{4 x}=1.37 \cos ^{2} \theta$,

$F_{1 y}=F_{3 i y}=-2.00 \sin \theta \cos \theta$,

and

$F_{2 y}=F_{4^{\prime} y}=2.00 \sin \theta \cos \theta$.

Fig. 3 shiows the radial and tangential forces through a complete current cycle. We draw the following conclusion about the behavior of the conductors:

- Torques caused by the tangential forces about the central Z-axis cancel, hence there is no tendency for the circuit to rotate about the Z-axis.

- There is no radially inward force on any conductor during the entire cycle.

- Pedk radial outward force $\approx 8.0 \mathrm{lb} / \mathrm{in}$ on each conductor.

- Peák tangential force $\approx 5.2 \mathrm{lb} /$ in on each conductor. 


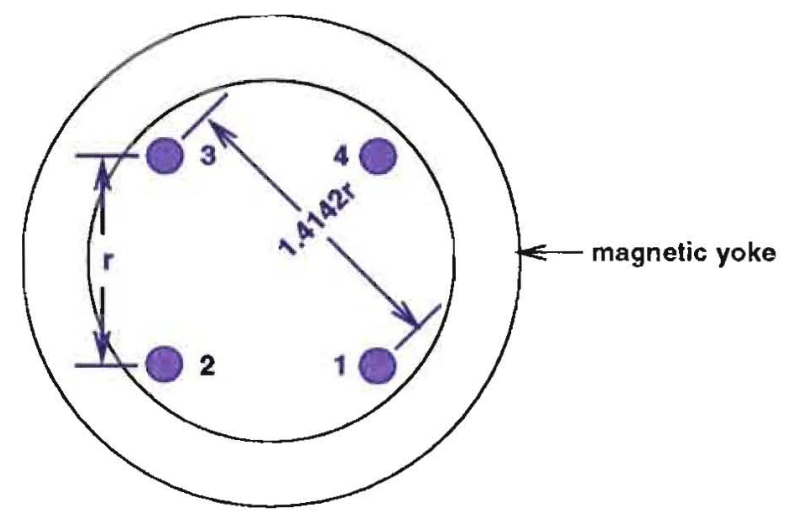

Figure 1: Cross section of conductors; $\mathrm{r}$ is $2 \mathrm{~cm}$.

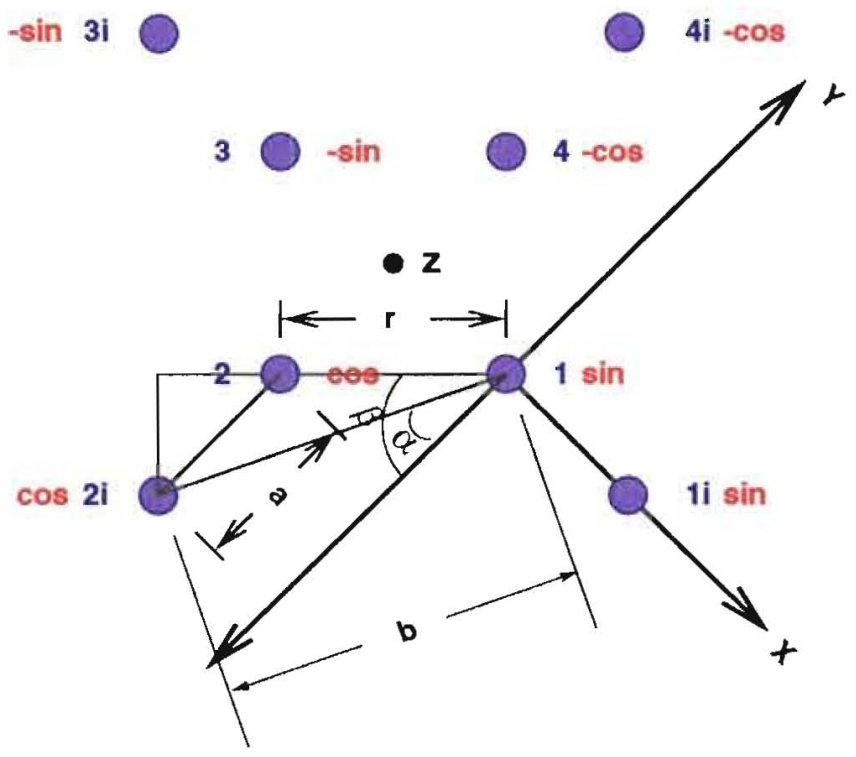

Figure 2: Equivalent current distribution in which the magnetic shield $(\mu=\infty)$ is replaced with 4 image curients. 


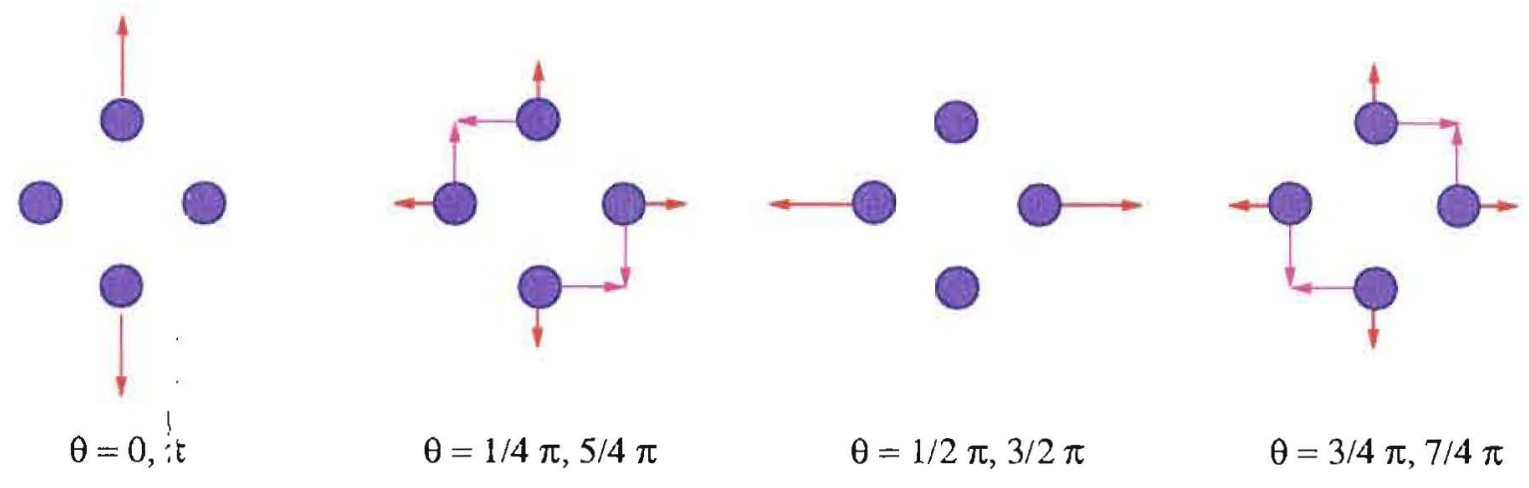

Figure 3: The forces on conductors during a full cycle of current pulse. 